\title{
COMPUTATION ON CONFORMAL MODULI OF
} QUADRILATERALS

\section{loane Shengelia*}

Tbilisi State University,

I Ilia Chavchavadze Avenue, Tbilisi, Georgia

E-mail: Ioane_shengelia@mail.ru

In this paper we consider classification of general topological quadrilaterals by conformal moduli and give one possible method of the numerical computation of this conformal invariant of quadrilaterals by elliptic integral of the first kind. We clarify relation between conformal moduli and AGM and give some examples.

RDP online workshop "Recent Advances in Mathematical Physics" - Regio2020,

5-6 December 2020

online

* Speaker 


\section{Introduction}

Investigation of conformal moduli of quadrilaterals plays an important role in geometric function theory and have remarkable physical interpretation. In recent years, a lot of attention has been paid to numerical computation of conformal moduli of some classes of quadrilaterals such as those associated with polygonal domains or domains bounded by circular arcs, because the last is used for study many mathematical and physical problems, in particular, it is powerful tool for investigation boundary value of analytic functions and quasiconformal mappings (see [10], [3]), configuration space of planar and spherical linkages [6], electrostatic problems (see an example 5.9.5 in [1]), and so on.

There exist some different methods for calculation of moduli of quadrilaterals. For example to find the moduli in [2] is solved the Dirichlet-Neumann boundary value problem for the Laplace equation. Modern numerical approach to problem and overview such type methods considered in recently monograph [8]. Namely, in [8] is considered the numerical method based on Schwarz ODE, in particular, in first found the accessory parameters of equation and next, solved the equation and to computed the moduli of the quadrilateral.

Here we give weak modification of the methods given in [8], [9], based on elliptic integral of the first kind and give interesting relation between conformal moduli of quadrilaterals and arithmetic geometric mean AGM.

Before we consider the conpect of the moduli, it is advisable to establish some mathematical definitions and results. First of all, lets consider the conformal maps. Let $C$ be a complex plane. A function $f: C \rightarrow C$ is said to be conformal, if it is analytic and localy injection. It is well-known, that the conformality is an equivalence relation between some subsets of the complex plane. Indeed, the inverse of the conformal map is also conformal, also, the composition of the conformal maps is also conformal. This implies the symmetric and transition properties of the conformal equivalence relation between concrete subsets of the complex plane.

The first example of conformal equivalence is the equivalence of the simple-connected domains. The simple-connected domain is such subset of the complex plane, for which all closed curves in it are null-homotopic.

Riemann Mapping Theorem. Let $D$ be a simple-connected domain which is a proper subset of the complex place (but not the whole complex plane). Then there exists bijective conformal map from $D$ to the unit disk $\Delta=\{z \in C:|z|<1 \mid\}[1]$.

By the inverse and transitive properties of conformal map it is clear that all simple-connected domains are conformally equivalent. Now lets consider the Jordan domains. The Jordan curve is such curve on the complex plane, which is 1-1 continuous image of $R / Z$. By the Jordan curve theorem [7], the Jordan curve divides the complex plane into two domains with the common boundary $\Gamma$ - the interior (or bounded domain) which is called a Jordan Domain, and the exterior (or unbounded domain) of the curve.

Caratheodory Extension Theorem. Let $D$ be a Jordan domain bounded by a Jordan curve $\Gamma$ and let $f$ be some conformal map from $D$ into the unit disk $\Delta$. Then $f$ can be extended to a homeomorphism (1-1 continuous map) of $\bar{D}=D \cup \Gamma$ into the closed disk $\bar{\triangle}$ [4].

Likewise the simple-connected domains, it is clear, that all Jordan domains are conformally equivalent to each other. One can consider the triangles. The triangle is the Jordan domain with 
fixed three points on its boundary. Two triangles are called conformally equivalent if the conformal map between them maps the three points from the first triangle into the three points of the other triangle. It is proven, that all triangles are conformally equivalent to each other.

Thus, all simple-connected domains are conformally equivalent. Also all Jordan domains are conformally equivalent. Likewise all triangles are conformally equivalent. What about the quadrilaterals? - we will consider the problem of the conformal classification of the quadrilaterals at the following section.

\section{Conformal classification of quadrilaterals}

Let $Q$ be some Jordan domain on the complex plane and $\Gamma$ be it's boundary. Fix four distinct points on the boundary $\left\{q_{1}, q_{2}, q_{3}, q_{4}\right\} \in \Gamma$ (with the positive orientation). The Jordan domain with fixed four points on its boundary is called the quadrilateral. Suppose $Q\left(q_{1}, q_{2}, q_{3}, q_{4}\right)$ and $Q^{\prime}\left(q_{1}^{\prime}, q_{2}^{\prime}, q_{3}^{\prime}, q_{4}^{\prime}\right)$ are two quadrilaterals. They are said to be conformally equivalent to each other, if there exists an orientation preserving conformal map $f: Q \rightarrow Q^{\prime}$ such that

$$
f:\left\{q_{1}, q_{2}, q_{3}, q_{4}\right\} \rightarrow\left\{q_{1}^{\prime}, q_{2}^{\prime}, q_{3}^{\prime}, q_{4}^{\prime}\right\} .
$$

Lets denote the set of conformal equivalence classes by $\{Q / C\}$. Suppose $M$ is some set. It is called the set of conformal invariants of the quadrilaterals if its is bijective to $\{Q / C\}$. In other words, the set of conformal invariants is something like representation of the classes. Let me consider an example of it.

Let $Q\left(q_{1}, q_{2}, q_{3}, q_{4}\right)$ be some quadrilateral with the boundary $\Gamma$. Suppose $h(Q)$ is conformal mapping into the upper half plane which maps the boundary of $Q$ into the real line and the four points into $\left\{x_{1}, x_{2}, x_{3}, x_{4}\right\}$. It is well-known, that the function

$$
f(z)=\frac{z-x_{1}}{z-x_{4}} \frac{x_{2}-x_{4}}{x_{2}-x_{1}}
$$

maps the upper half plane into itself, such that the three points - $\left\{x_{1}, x_{2}, x_{4}\right\}$ are mapped into the three points $-\{0,1, \infty\}$. As for $x_{3}$ :

$$
f\left(x_{3}\right)=\xi\left(x_{1}, x_{2}, x_{3}, x_{4}\right)=\frac{x_{3}-x_{1}}{x_{3}-x_{4}} \frac{x_{2}-x_{4}}{x_{2}-x_{1}}>1,
$$

which is called the cross-ratio of the four points.

Theorem. $\xi$ is conformal invariant of quadrilaterals.

Proof. First of all, let me prove that $\xi$ has different values for the different classes of conformal equivalence. Indeed, suppose $Q$ and $Q^{\prime}$ are some quadrilaterals. Suppose their corresponding value of $\xi$ is the same. Then by the transition property of conformal map there must be conformal map between $Q$ and $Q^{\prime}$ :

$$
\left\{q_{1}, q_{2}, q_{3}, q_{4}\right\} \rightarrow\{0,1, \xi, \infty\} \rightarrow\left\{q_{1}^{\prime}, q_{2}^{\prime}, q_{3}^{\prime}, q_{4}^{\prime}\right\}
$$

Therefore, if two quadrilaterals are not conformally equivalent, then their corresponding values of $\xi$ are different. 
Secondly, we prove, that $\xi$ has the same value for the conformally equivalent quadrilaterals. Indeed, suppose $Q$ is some quadrilateral. Let $h(Q)$ be conformal map into the upper half plane. Thus there are four fixed points $\left\{x_{1}, x_{2}, x_{3}, x_{4}\right\}$ and the corresponding value $\xi$. Let $Q^{\prime}$ be conformally equivalent to $Q$. Let $h^{\prime}\left(Q^{\prime}\right)$ be conformal map into the upper half plane with the corresponding four fixed points $\left\{x_{1}^{\prime}, x_{2}^{\prime}, x_{3}^{\prime}, x_{4}^{\prime}\right\}$ and $\xi^{\prime}$.

By the transition property of conformal map there must be conformal mapping from the upper half plane with four points $\left\{x_{1}, x_{2}, x_{3}, x_{4}\right\}$ into the upper half plane with four points $\left\{x_{1}^{\prime}, x_{2}^{\prime}, x_{3}^{\prime}, x_{4}^{\prime}\right\}$. It is well-known that any automorphism of the upper half plane is a bilinear transformation

$$
g(z)=\frac{a z+b}{c z+d} .
$$

But it is also well known that $\xi$ parameter is invariant under bilinear maps. Therefore $\xi=\xi^{\prime}$. From this follows, that $\xi$ is uniquely defined for conformally equivalent quadrilaterals. Therefore, there is bijection between the set of the values of $\xi$ and $\{Q / C\}$.

Using (1) we map the upper half plane into itself such that the image of the four points $\{0,1, \xi, \infty\}$ will be $\{-\eta,-1,1, \eta\}$, where

$$
\eta=\frac{\sqrt{\xi}+1}{\sqrt{\xi}-1}>1
$$

Suppose $k=1 / \eta$. Then $k$ is also conformal invariant. Indeed, there exists one-to-one relation between $k$ and $\xi$, because $k$ is monotonic function of $\xi$ and vice versa:

$$
\frac{d k}{d \xi}=\frac{1}{\sqrt{\xi}(\sqrt{\xi}+1)^{2}}>0 .
$$

When $\xi$ varies in $(1, \infty)$, then $k$ monotonously varies in $(0,1)$. Therefore, two quadrilaterals are conformally equivalent iff their corresponding values of $\xi$ or $k$ are the same.

Now we characterise the analytic properties of the moduli of quadrilaterals. There exists conformal map from the upper half plane into the rectangle, called the schwarz-christoffel transformation, which maps the real line into the boundary of the rectangle and the four points $-\{-\eta,-1,1, \eta\}$ into the vertices of the rectangle - $\left[D_{x}, D_{x}+i D_{y},-D_{x}+i D_{y},-D_{x}\right]$, where $D_{x}$ and $D_{y}$ are expressed by the elliptic integrals [9]. The moduli of such rectangle is ratio of two line segments of it:

$$
\mu(k)=\frac{2 B k K(k)}{B k K\left(k^{\prime}\right)}=2 \frac{\int_{0}^{1} \frac{d \omega}{\sqrt{1-\omega^{2}} \sqrt{1-k^{2} \omega^{2}}}}{\int_{0}^{1} \frac{d \omega}{\sqrt{1-\omega^{2}} \sqrt{1-k^{\prime 2} \omega^{2}}}},
$$

where $k^{\prime}=\sqrt{1-k^{2}}<1$.

Proposition. The moduli of the quadrilaterals is conformal invariant.

Proof. There exists one-to-one relation between $\mu$ and $k$. Indeed $\mu$ is monotonic function of $k$, because the derivative of $\mu$ by $k$

$$
2 k \frac{\int_{0}^{1} \frac{d \omega}{\sqrt{1-\omega^{2}}\left(1-k^{2} \omega^{2}\right)^{3 / 2}} \int_{0}^{1} \frac{d \omega}{\sqrt{1-\omega^{2}} \sqrt{1-k^{\prime 2} \omega^{2}}}+\int_{0}^{1} \frac{d \omega}{\sqrt{1-\omega^{2}} \sqrt{1-k^{2} \omega^{2}}} \int_{0}^{1} \frac{d \omega}{\sqrt{1-\omega^{2}}\left(1-k^{\prime 2} \omega^{2}\right)^{3 / 2}}}{\left[\int_{0}^{1} \frac{d \omega}{\sqrt{1-\omega^{2}} \sqrt{1-k^{\prime 2} \omega^{2}}}\right]^{2}}
$$


is always positive.

From the above follows, that each parameter from the triple $\{\xi, k, \mu\}$ is monotonic function of the others and they all are representations of $\{Q / C\}$.

Corollary. Two rectangles are conformaly equivalent iff they are similar.

Suppose, one of the four points on the boundary of $Q$ is moving. We are showing how the moduli varies at these cases. As we showed before, $\frac{d \mu}{d k}$ and $\frac{d k}{d \xi}$ are always positive. So, the sine of $\frac{\partial \mu}{\partial x_{i}}$ is determined by the sine of $\frac{\partial \xi}{\partial x_{i}}$, because

$$
\frac{\partial \mu}{\partial x_{i}}=\frac{d \mu}{d k} \frac{d k}{d \xi} \frac{\partial \xi}{\partial x_{i}} .
$$

Note, that for each $Q\left[q_{1}, q_{2}, q_{3}, q_{4}\right]$ quadrilateral there exists the orientation preserving conformal mapping into the upper half plane which has a 1-1 continuous extension between the boundary of $Q$ and the real line. Therefore, the character of "increasement/decreasement" of moduli determined by the motions of the four points $x_{i}$ on the real line is the same as the character determined by the motion of the corresponding points $q_{i}$ on the boundary of $Q$. So, we get the following relations

$$
\begin{gathered}
\frac{\partial \xi}{\partial x_{1}}=\frac{x_{2}-x_{4}}{x_{3}-x_{4}} \frac{x_{3}-x_{2}}{\left(x_{2}-x_{1}\right)^{2}}>0 \Longleftrightarrow \frac{\partial \mu}{\partial x_{1}}>0 \Rightarrow \lim _{q_{1} \rightarrow q_{2}} \mu=\infty, \\
\frac{\partial \xi}{\partial x_{1}}=\frac{x_{2}-x_{4}}{x_{3}-x_{4}} \frac{x_{3}-x_{2}}{\left(x_{2}-x_{1}\right)^{2}}>0 \Longleftrightarrow \frac{\partial \mu}{\partial x_{2}}<0 \Rightarrow \lim _{q_{2} \rightarrow q_{3}} \mu=0, \\
\frac{\partial \xi}{\partial x_{1}}=\frac{x_{2}-x_{4}}{x_{3}-x_{4}} \frac{x_{3}-x_{2}}{\left(x_{2}-x_{1}\right)^{2}}>0 \Longleftrightarrow \frac{\partial \mu}{\partial x_{3}}>0 \Rightarrow \lim _{q_{3} \rightarrow q_{4}} \mu=\infty, \\
\frac{\partial \xi}{\partial x_{1}}=\frac{x_{2}-x_{4}}{x_{3}-x_{4}} \frac{x_{3}-x_{2}}{\left(x_{2}-x_{1}\right)^{2}}>0 \Longleftrightarrow \frac{\partial \mu}{\partial x_{4}}<0 \Rightarrow \lim _{q_{4} \rightarrow q_{1}} \mu=0 .
\end{gathered}
$$

\section{Description of the method of numerical computation}

Suppose, it is known the value of $\xi$ parameter of some quadrilateral. As for the moduli, the function $\mu(\xi)$ is not represented analytically. But the properties of $\mu$ allows us to find some discrete values of the pair of conformal invariants $(\xi, \mu)$. The moduli has the following properties:

1. $\mu(k) \mu\left(k^{\prime}\right)=4$ (by (3) formula)

2. $\mu\left(q_{1}, q_{2}, q_{3}, q_{4}\right) \mu\left(q_{2}, q_{3}, q_{4}, q_{1}\right)=1$

3. $\mu\left(q_{1}, q_{2}, q_{3}, q_{4}\right)=\mu\left(q_{3}, q_{4}, q_{1}, q_{2}\right)$

by the rectangle properties. Therefore, for some known value of $\mu$ one can find two other values of moduli by the first and second properties. But we are interested in how the other conformal invariants such as $k$ and $\xi$ are changed.

Using the first property. Suppose we have found some value of moduli $\mu$ for some $k$ and $\xi$. By the first property, if change $k$ to $k^{\prime}=\sqrt{1-k^{2}}$ then the moduli will be changed as follows

$$
\mu\left(k^{\prime}\right)=\frac{4}{\mu(k)} .
$$


As for $\xi$

$$
\xi=\left(\frac{1+k}{1-k}\right)^{2} \rightarrow \xi^{\prime}=\left(\frac{1+k^{\prime}}{1-k^{\prime}}\right)^{2}=\left(\frac{1+\sqrt{1-k^{2}}}{1-\sqrt{1-k^{2}}}\right)^{2} .
$$

So the triple of conformal invariants will be transformed by the rule

$$
[\mu, k, \xi] \rightarrow_{k}\left[\frac{4}{\mu}, \sqrt{1-k^{2}},\left(\frac{1+\sqrt{1-k^{2}}}{1-\sqrt{1-k^{2}}}\right)^{2}\right] .
$$

Lets call this K-transformation and note it by $\rightarrow_{k}$.

Using the second property. Suppose we have found some value of moduli $\mu$ for some $k$ and $\xi$. By the second property if permutate the four points $\left\{q_{1}, q_{2}, q_{3}, q_{4}\right\}$ into $\left\{q_{2}, q_{3}, q_{4}, q_{1}\right\}$ then the moduli will be changed as follows

$$
\mu\left(q_{2}, q_{3}, q_{4}, q_{1}\right)=\frac{1}{\mu\left(q_{1}, q_{2}, q_{3}, q_{4}\right)} .
$$

As for $\xi$ and $k$, we get the transformation formulas by (2). Suppose

$$
\begin{aligned}
\xi\left(x_{1}, x_{2}, x_{3}, x_{4}\right) & =\frac{x_{3}-x_{1}}{x_{3}-x_{4}} \frac{x_{2}-x_{4}}{x_{2}-x_{1}}, \\
\xi^{\prime}\left(x_{1}, x_{4}, x_{3}, x_{2}\right) & =\frac{x_{3}-x_{1}}{x_{3}-x_{2}} \frac{x_{4}-x_{2}}{x_{4}-x_{1}} .
\end{aligned}
$$

We need the equation, which represents the relation between $\xi$ and $\xi^{\prime}$. Suppose $\left\{x_{1}, x_{2}, x_{3}, x_{4}\right\}$ are four fixed points on the real line corresponding to some quadrilateral. By (1) mapping (which is conformal) and transitive property of conformal mappings there are conformally equivalent four points $\{-1,0,1, q\}$ where $q>1$ depends on $\left\{x_{1}, x_{2}, x_{3}, x_{4}\right\}$ or more specifically on $\xi$. As the conformal maps between the upper half planes are bilinear maps and $\xi$ is invariant under such maps, the following identities

$$
\xi=\frac{1-(-1)}{1-q} \frac{0-q}{0-(-1)}=\frac{2 q}{q-1} \Rightarrow q=\frac{\xi}{\xi-2}
$$

holds. Suppose we permutate the four points $\left\{x_{1}, x_{2}, x_{3}, x_{4}\right\}$ into $\left\{x_{2}, x_{3}, x_{4}, x_{1}\right\}$. By the conformal equivalence of $\left\{x_{1}, x_{2}, x_{3}, x_{4}\right\}$ and $\{-1,0,1, q\}$ it means that we changed it into $\{0,1, q,-1\}$. Thus

$$
\xi^{\prime}=\frac{q-0}{q-(-1)} \frac{1-(-1)}{1-0}=\frac{2 q}{q+1},
$$

but $q=\xi /(\xi-2)$, i.e. we get the following transformation formula for $\xi$

$$
\xi^{\prime}=\frac{\xi}{\xi-1}
$$

As for $k$, it is changed by the relation

$$
k=\frac{\sqrt{\xi}-1}{\sqrt{\xi}+1} \Rightarrow \frac{\sqrt{\xi}-\sqrt{\xi-1}}{\sqrt{\xi}+\sqrt{\xi-1}} .
$$


So, $\xi$ is transformed like (5) and the triple of conformal invariants will be transformed as follows

$$
[\mu, k, \xi] \rightarrow \xi\left[\frac{1}{\mu}, \frac{\sqrt{\xi}-\sqrt{\xi-1}}{\sqrt{\xi}+\sqrt{\xi-1}}, \frac{\xi}{\xi-1}\right] .
$$

Lets call this $\xi$-transformation and note it by $\rightarrow \xi$.

Thus for each value of moduli one can find two other values of moduli and corresponding $k$ and $\xi$ parameters by the formulas (4) and (6).

Remark. Note that both $\mathrm{K}$ and $\xi$ transformations are symmetric:

$$
\begin{aligned}
& \mu \rightarrow_{k} \frac{4}{\mu} \rightarrow_{k} \frac{4}{4 / \mu}=\mu \Rightarrow \mu \leftrightarrow_{k} \frac{4}{\mu}, \\
& \mu \rightarrow_{\xi} \frac{1}{\mu} \rightarrow_{\xi} \frac{1}{1 / \mu}=\mu \Rightarrow \mu \leftrightarrow_{\xi} \frac{1}{\mu} .
\end{aligned}
$$

We can make some nonsymmetric combinations

$$
\ldots \leftrightarrow_{k} \frac{1}{4 \mu} \leftrightarrow_{\xi} 4 \mu \leftrightarrow_{k} \frac{1}{\mu} \leftrightarrow_{\xi} \mu \leftrightarrow_{k} \frac{4}{\mu} \leftrightarrow_{\xi} \frac{\mu}{4} \leftrightarrow_{k} \frac{16}{\mu} \leftrightarrow_{\xi} \ldots
$$

and there are no other members made by this transformations except $2^{2 s} \mu$ and $2^{2 s} \mu^{-1}$, where $s$ is an integer. For all $s \in Z$ one can find corresponding $k$ and $\xi$ by the reccurent formulas (4) and (6). Also note, that the chain made by the each member of the chain (7) is the same chain.

The particular case. By (4) formula it is clear, that if $k=k^{\prime}=1 / \sqrt{2}$ then $\mu=2$. Thus one can calculate all members of the corresponding chain. But let me generalize a little this method. Suppose

$$
2^{2 s^{\prime}} \mu=\frac{2^{2 s^{\prime \prime}}}{\mu},
$$

this implies $-\mu=2^{s}$. We get two chains corresponding to "odd" and "even" $s$

$$
\begin{gathered}
2 \leftrightarrow_{\xi} \frac{1}{2} \leftrightarrow_{k} 8 \leftrightarrow_{\xi} \frac{1}{8} \leftrightarrow_{k} 32 \leftrightarrow_{\xi} \frac{1}{32} \leftrightarrow_{k} 128 \leftrightarrow_{\xi} \frac{1}{128} \ldots, \\
1 \leftrightarrow_{k} 4 \leftrightarrow_{\xi} \frac{1}{4} \leftrightarrow_{k} 16 \leftrightarrow_{\xi} \frac{1}{16} \leftrightarrow_{k} 64 \leftrightarrow_{\xi} \frac{1}{64} \ldots .
\end{gathered}
$$

respectively.

For the "odd chain" we know that if $\mu=2$, then $k=1 / \sqrt{2}$ and so the triple of conformal invariants is:

$$
[2,0.707106781187,33.9705627485] \text {. }
$$

As for the "even chain", note, that $1 \leftrightarrow \xi 1$. By the 1-1 relation between the conformal invariants, the same moduli implies the same values of other conformal invariants. Therefore, by (5), $\xi=\xi^{\prime}=2$. So the triple is

$$
[1,0.171572875254,2] \text {. }
$$

Thus, we know the first values of conformal invariants and by the reccurent formulas (4) and (6) we are able to get an infinite number of members of the both chains. We represent the discrete graph of function $\mu(k)$ at the following figure. 
Figure 1: The discretre graphic of $\mu(k)$

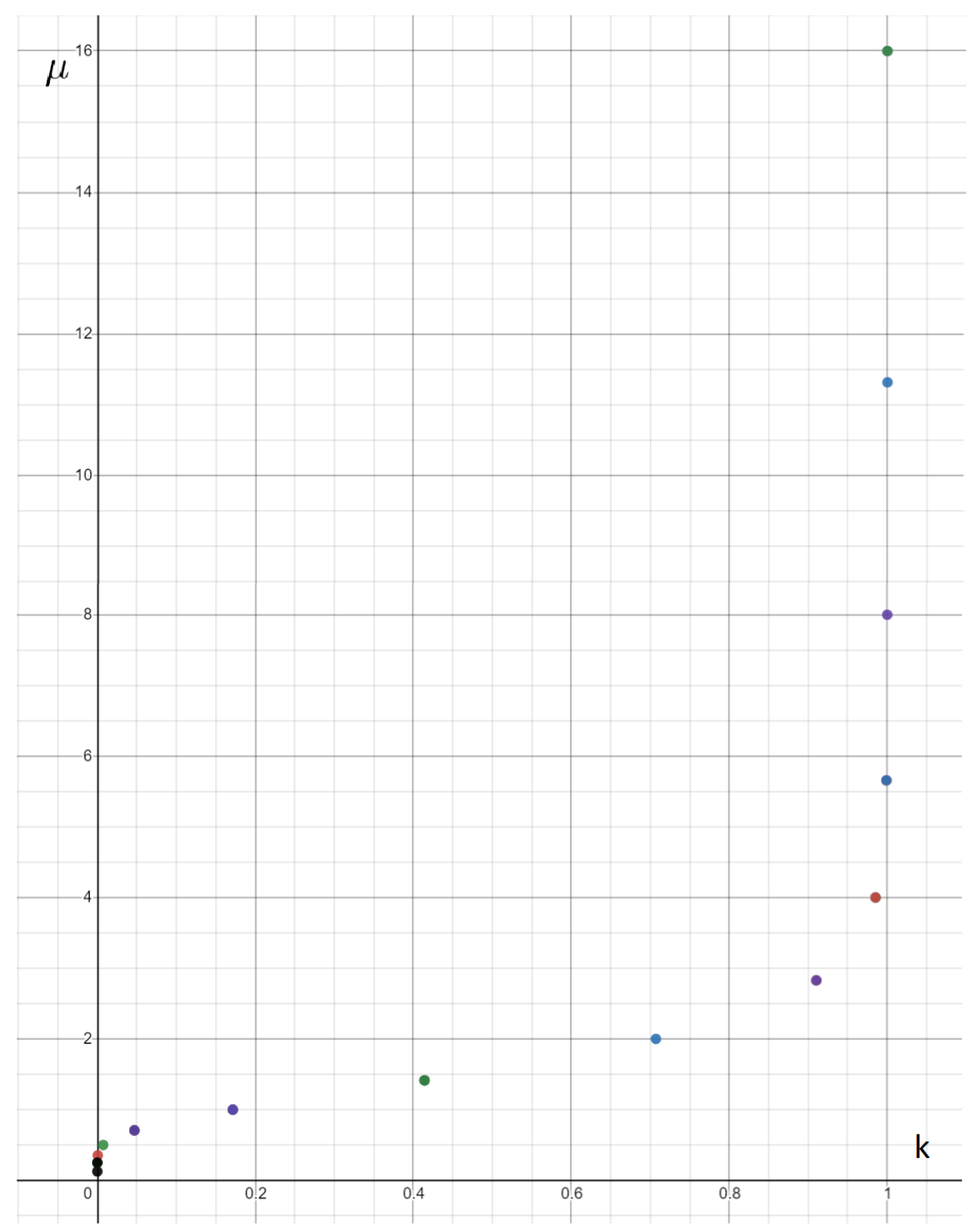

\section{Relation to arithmetic-geometric mean}

Let $a_{0}$ and $b_{0}$ be the real numbers and $a_{0} \geq b_{0}>0$. Define two sequences

$$
a_{n}=\frac{a_{n-1}+b_{n-1}}{2}, b_{n}=\sqrt{a_{n-1} b_{n-1}} .
$$

It is well known that the limits of these two sequences are the same,

$$
M\left(a_{0}, b_{0}\right)=\lim _{n \rightarrow \infty} a_{n}=\lim _{n \rightarrow \infty} b_{n} .
$$

Such limit is called the arithmetic-geometric mean of the numbers $\left(a_{0}, b_{0}\right)$ (noted by AGM). 
Let's define the inverse sequence

$$
a_{n-1}=a_{n}+\sqrt{a_{n}^{2}-b_{n}^{2}}, b_{n-1}=a_{n}-\sqrt{a_{n}^{2}-b_{n}^{2}} .
$$

AGM has the following properties:

1. bilinearity: $M(\rho a, \rho b)=\rho M(a, b)$.

2. symmetry: $M(a, b)=M(b, a)$.

3. sequence invariance:

$$
\ldots=M\left(a_{-2}, b_{-2}\right)=M\left(a_{-1}, b_{-1}\right)=M\left(a_{0}, b_{0}\right)=M\left(a_{1}, b_{1}\right)=M\left(a_{2}, b_{2}\right)=\ldots
$$

It is well known that the relation of the elliptic integrals and AGM is the following identity [5].

$$
K(k)=\frac{\pi / 2}{M\left(1, \sqrt{1-k^{2}}\right)} .
$$

Therefore, by (3) the moduli can be written as

$$
\mu(k)=2 \frac{M(1, k)}{M\left(1, \sqrt{1-k^{2}}\right)} .
$$

Consider the case, when $a=1$ and $b=k<1$. Using the properties of AGM, we obtain

$$
\ldots=v_{-1}(k) M\left(1, \tau_{-1}(k)\right)=v_{0}(k) M\left(1, \tau_{0}(k)\right)=v_{1}(k) M\left(1, \tau_{1}(k)\right)=\ldots,
$$

where the coefficients are calculated reccurently

$$
\begin{gathered}
\tau_{0}(k)=k ; \tau_{d}(k)=\frac{2 \sqrt{\tau_{d-1}(k)}}{1+\tau_{d-1}(k)}, \tau_{-d}(k)=\frac{1-\sqrt{1-\tau_{-d+1}^{2}(k)}}{1+\sqrt{1-\tau_{-d+1}^{2}(k)}}, \\
v_{0}(k)=1 ; v_{d}(k)=\prod_{i=0}^{d-1} \frac{1+\tau_{i}(k)}{2}, v_{-d}(k)=\prod_{j=-d+1}^{0}\left(1+\sqrt{1-\tau_{j}^{2}(k)}\right) .
\end{gathered}
$$

So, using (8) and the reccurent formulas, the moduli can be written as

$$
\mu_{s}=2 \frac{M(1, k)}{M\left(1, \tau_{s}(k)\right)}=2 v_{s}(k),
$$

where $k$ must be the solution of the following equation:

$$
\sqrt{1-k^{2}}=\tau_{s}(k)
$$

Thus, it is possible to calculate the moduli for some discrete values of $k$.

Proposition. for each $s \in Z$ the equation (10) always has one solution.

Indeed, for $s=0, \sqrt{1-k^{2}}=k$. So there exists one solution corresponding to intersection point of the circle and line. For $s= \pm 1$ it is clear that if $k$ varies from 0 to 1 then $\tau_{ \pm 1}(k)$ also 
varies (monotonously) from 0 to 1 . For other cases of $s \in Z$ we must put the parameter with the same interval $(0,1)$ at the same function. Thus all functions $\tau_{s}(k)$ for all $s$ monotonously increases from 0 to 1 , however the velocity of their increasement is different for every $s$. As for the function $\sqrt{1-k^{2}}$, it monotonously decreases from 1 to 0 at the same time. So, they must intersect each other only one times and the intersection point will be different for each $s$. Thus, the equation (10) has one solution for each $s$.

\section{Conclusion}

Using the method above, for all known values of conformal invariants, which are calculated by (9) and (10), it is possible to build an infinity chains of other values using the reccurent formulas (4) and (6). Also, for every known values of conformal invariants, which are calculated by the other methods, it is possible to build chains of discrete values by the formulas (4) and (6).

Acknowledgement. This work was supported by the joint grant of Volkswagen Foundation and Shota Rustaveli National Science Foundation of Georgia (Ref. 93562 \& \# 04/48).

\section{References}

[1] M. Ablowitz., S. Focas. Complex Variables. Introduction and Applications. Second edition. Cambridge texts in applied mathematics. 2003.

[2] L.V. Ahlfors. Conformal invariants: Topics in Geometric Function Theory. McGraw-Hill, New York, 1973.

[3] K. Astala, T. Iwaniec, G. Martin. Elliptic partial differential equations and quasiconformal mappings in the p lane. Princeton Mathematical Series 18, 2009.

[4] J. Bak, J. Newman. Complex Analysis, Third Edition. Springer. Undergraduate texts in mathematics. 2017.

[5] D.A. Cox. Gauss and the Arithmetic-Geometric Mean. Department of Mathematics and Statistics. Amherst College. CTNT, 2016.

[6] G.Giorgadze, G. Khimshiashvili. Cyclic configurations of spherical quadrilaterals. Bull.Georgian Nat.Acag.Sci. 3 (2), 23-27, 2009.

[7] T. Hales. Jordan's Proof of the Jordan Curve Theorem. Students in Logic, Grammar and Rhetoric 10 (23) 2007.

[8] P. Hariri, R. Klen and M. Vuorinen. Conformally Invariant Metrics and Quasiconformal Mappings. Springer, Springer Monographs in Mathematics, 2020.

[9] I. Shengelia. Conformal modulus of quadrilaterals. Proceedings of I. Vekua Institute of Applied Mathematics Vol. 67, 2017.

[10] A.R. Tsitskishvili. Effective methods for solving the problems of conformal mapping and the theory of filtration. Diss. Doct. Phys.-Math. Sci., Moscow, 1981. 